\section{OP50 STAKEHOLDER FRAMING OF ADVERTISING LEGISLATION: AN ANALYSIS OF MEDIA AND PARLIAMENTARY REPRESENTATIONS OF THE LOI ÉVIN IN THE UK}

${ }^{1} \mathrm{~N}$ Maani Hessari ${ }^{*},{ }^{1} \mathrm{C}$ Knai, ${ }^{2} \mathrm{~K}$ Gallopel-Morvan, ${ }^{3} \mathrm{M}$ Petticrew, ${ }^{4} \mathrm{M}$ Guillou Landreat. ${ }^{1}$ Department of Health Services Research and Policy, London School of Hygiene and Tropical Medicine, London, UK; ${ }^{2}$ EHESP, School of Public Health, Rennes, France; ${ }^{3}$ Department of Public Health, Environments and Society, London School of Hygiene and Tropical Medicine, London, UK; ${ }^{4}$ Addictive Disorders Department, Université de Bretagne Occidentale, Brest, France

\subsection{6/jech-2018-SSMabstracts.50}

Background The Loi Évin legislation restricts alcohol advertising in France, and is more comprehensive than the self-regulation-based UK approach. This project aimed to analyse how the Loi Évin has been represented in UK media and parliament by the advertising and alcohol industries, politicians and non-governmental organisations. It is important to understand this because it can potentially shed light on how stakeholders, including industry, frame the debate around public health policy.

Methods Qualitative analysis of media and parliamentary documents using the hermeneutic method, assessing contexts in which the Loi Évin was mentioned by stakeholders in the media and in parliament. Print and trade media articles referencing the Loi Évin were retrieved from the PROQUEST media archive [from 1985-2016]. UK parliamentary representations referencing the Loi Évin were obtained from the parliamentary database for publications and the Hansard parliamentary record. In total, 109 documents referencing the Loi Évin were identified, of which 71 (44 articles from ProQuest, 27 from parliamentary sources) contained direct quotes from stakeholders and were therefore included. These were analysed thematically using the hermeneutic method, which involved reading and understanding meanings of individual texts, in this case the contexts in which the Loi Évin was mentioned, identifying sub-themes or 'codes', grouping these into broader thematic clusters of codes, triangulating findings between different sources (for example, identifying similar arguments in print media and evidence submissions), assessing the reliability/validity of findings (through verification of coding by a second researcher), and illustrative use of representative case material. All coding was performed using NVivo 11.3.2.

Results The alcohol and advertising industries have framed the Loi Évin as incompatible with EU principles, irrational and ineffective, with their arguments changing over time in response to landmark events and rulings. Supporters of Loi Évin-style legislation by contrast failed to counter industry framing of arguments around the link between regulations and overall consumption changes.

Conclusion The portrayal of the Loi Évin by industry in the UK is an important case study of policy framing, and provides evidence of the synergy between the advertising and alcohol industries in combatting legislation that could potentially harm profit. Public health professionals practitioners, academics and advocates should be aware of the nature of such industry arguments when considering submissions to policy development processes.

\title{
Pregnancy and health
}

\section{OP51 OUTCOMES OF TWIN PREGNANCIES RECEIVING ANTENATAL CORTICOSTEROIDS: ANALYSIS OF ROUTINELY COLLECTED OBSTETRIC DATA}

${ }^{1}$ AA Ayorinde* , ${ }^{2}$ EA Raja, ${ }^{3}$ A Shetty, ${ }^{3} \mathrm{PJ}$ Danielian, ${ }^{2} \mathrm{~S}$ Bhattacharya. ${ }^{1}$ Warwick Centre for Applied Health Research and Delivery, University of Warwick, Coventry, UK; ${ }^{2}$ Dugald Baird Centre for Research on Women's Health, University of Aberdeen, Aberdeen, UK; ${ }^{3}$ Aberdeen Maternity Hospital, NHS Grampian, Aberdeen, UK

\subsection{6/jech-2018-SSMabstracts.51}

Background Antenatal corticosteroids (ACS) have been shown to reduce neonatal morbidity and mortality associated with preterm birth in singletons, particularly respiratory distress syndrome (RDS) and intraventricular haemorrhage. The evidence regarding the beneficial effects of ACS in twin pregnancies is less clear. The objective of this study was to assess the effect of ACS on neonatal mortality and morbidity in twins at risk of preterm ( $<37$ weeks gestation) birth.

Methods A cohort study design was used to analyse anonymised routinely collected data on all twin deliveries between 1996 and 2014 as recorded in the Aberdeen Maternity and Neonatal Databank. Those who received ACS (dexamethasone or betamethasone) and those who did not were classified as exposed and unexposed respectively. Outcomes included: stillbirth, neonatal death, admission to neonatal intensive care unit, as well as specific neonatal events, namely, RDS, bronchopulmonary dysplasia, intraventricular haemorrhage, neonatal seizure and necrotising enterocolitis. A composite adverse outcome variable was created for neonates with one or more of the pre-specified neonatal events. Multilevel mixed-effect generalized linear models were used to evaluate effect of ACS on neonatal morbidity and mortality. Associations are presented as relative risks (RR) with 95\% Confidence Intervals (CI). Propensity score matched fixed effects models were used to account for confounding by indication.

Results The study included 3312 babies; 1686 babies whose mothers had received ACS and 1626 babies whose mothers had not. The propensity score matched fixed effects model showed that ACS administration was not significantly associated with RDS [RR 0.92 (95\% CI 0.67, 1.27)], bronchopulmonary dysplasia $[R R 1.05(0.78,1.41)]$ or the composite adverse neonatal outcome [RR $1.11(0.92,1.34)]$. ACS administration was associated with a reduction in still-birth rate [RR $0.46(0.30,0.69)]$ but associated with increased admission to neonatal unit [RR $1.23(1.07,1.40)]$, intraventricular haemorrhage $[R R 2.4(1.15,5.02)]$ and neonatal deaths [RR 3.15 $(1.34,7.39)]$.

Conclusion This study suggests that ACS (using the same administration protocols as for singleton pregnancy) is associated with a significant reduction in stillbirth rate in twins but with an increased rate of neonatal death, intraventricular haemorrhage and neonatal unit admission. There was no effect on the rates of RDS and bronchopulmonary dysplasia. It is possible that twin pregnancies respond to ACS differently from singletons. There is a need for a multi-centre randomised controlled trial to evaluate the effects of ACS in multiple pregnancies and to find the optimum dose and timing for this to be effective. 